\title{
Recent Advances in Mining Time Series Data
}

\author{
Eamonn Keogh \\ Department of Computer Science \& Engineering, \\ University of California, Riverside, USA \\ eamonn@cs.ucr.edu \\ http://www.cs.ucr.edu/ eamonn
}

Much of the world's supply of data is in the form of time series. Furthermore, as we shall see, many types of data can be meaningfully converted into "time series", including text, DNA, video, images etc. The last decade has seen an explosion of interest in mining time series data from the academic community. There has been significant work on algorithms to classify, cluster, segment, index, discover rules, visualize, and detect anomalies/novelties in time series.

In this talk I will summarize the latest advances in mining time series data, including:

- New representations of time series data.

- New algorithms/definitions.

- The migration from static problems to online problems.

- New areas and applications of time series data mining.

I will end the talk with a discussion of "what's left to do" in time series data mining.

\section{References}

1. E. Keogh. Exact indexing of dynamic time warping. In Proceedings of the 8th International Conference on Very Large Data Bases, pages 406-417, 2002.

2. E. Keogh and S. Kasetty. On the need for time series data mining benchmarks: A survey and empirical demonstration. In Proceedings of the 8th ACM SIGKDD International Conference on Knowledge Discovery and Data Mining, pages 102-111, 2002.

3. E. Keogh, J. Lin, and W. Truppel. Clustering of time series subsequences is meaningless: Implications for past and future research. In Proceedings of the 3rd IEEE International Conference on Data Mining, pages 115-122, 2003.

4. E. Keogh, S. Lonardi, and C. Ratanamahatana. Towards parameter-free data mining. In Proceedings of the tenth ACM SIGKDD International Conference on Knowledge Discovery and Data Mining, 2004.

5. C.A. Ratanamahatana and E. Keogh. Everything you know about dynamic time warping is wrong. In Proceedings of the Third Workshop on Mining Temporal and Sequential Data, in conjunction with the Tenth ACM SIGKDD International Conference on Knowledge Discovery and Data Mining (KDD-2004), 2004. 